\title{
ADAPTAÇÃO DA “THE RESILIENCE SCALE” PARA A POPULAÇÃO ADULTA PORTUGUESA ${ }^{1}$
}

\author{
Cláudia Alexandra Ferreira de Carvalho Ng Deep \\ Isabel Pereira
}

Resumo: Este artigo descreve a adaptação da "The Resilience Scale" para portugueses adultos. Procedeu-se à retradução e validação psicométrica. Fez-se pré-teste e reteste. Obteve-se equivalência linguística/conceptual e validação psicométrica com alpha de Cronbach 0,868 para 23 itens, sugerindo consistência interna. A análise fatorial agrupou 23 variáveis em 4 fatores: I - Perseverança; II - Sentido de vida; III - Serenidade; IV - Autossuficiência e autoconfiança, com valores alpha de Cronbach satisfatórios para cada fator. Verificou-se sensibilidade dos itens através do coeficiente de assimetria e achatamento e comprovou-se a estabilidade temporal. A escala revela boas características psicométricas aferindo níveis de resiliência nesta população.

Palavras-chave: Resiliência. Adaptação de testes (Portugal). Adultos.

1 Agradecimentos: Dra. Gail Wagnild, Dra. Cristiana Felgueiras, Instituto Superior Dom Afonso III, Instituto Superior de Psicologia Aplicada e Igreja Evangélica Batista de Faro. 


\section{Introdução}

A resiliência é um constructo psicológico altamente valorizado graças à sua estreita relação com a capacidade de adaptação ao meio e superação adequada de situações adversas. Resilir, em latim, significa literal e etimologicamente saltar para trás, retrair-se, sendo a palavra constituída pelo elemento re que indica um movimento para trás e pelo elemento salire ou saltar, "voltar a entrar saltando" ou "saltar para cima" (Felgueiras, 2008). Resiliência é um termo que habitualmente designa (na física) a resistência de um material a choques elevados e a sua capacidade para absorver a energia cinética do meio modificando-se, mas retornando posteriormente ao original, como o elástico que, após uma tensão inicial, volta ao mesmo estado. Na metalurgia encontra-se associado à capacidade de um material voltar ao estado inicial após pressões deformadoras e em informática refere-se à capacidade de um sistema continuar a funcionar apesar de falhas em seus elementos constitutivos. Em medicina remete para a capacidade de resistir a uma doença, não sendo, no entanto a simples capacidade de resistência (na ideia de rigidez), mas englobando a maleabilidade e a capacidade de adaptação. Em psicologia aponta para a capacidade de enfrentar positivamente acontecimentos adversos numa perspetiva de promoção da saúde, bem-estar e qualidade de vida. $O$ estudo desse constructo iniciou com a identificação das características psicológicas presentes em crianças capazes de viver e superar condições de vida adversas (Gonçalves, 2003), e estudos (Pesce, Assis, Avanci, Santos, Malaquias, \& Carvalhães, 2005) com crianças resilientes revelam 3 tipos de variáveis capazes de proteger o indivíduo na adversidade: características de personalidade (como autoestima, flexibilidade, habilidade para resolver conflitos), coesão e bom relacionamento familiar e disponibilidade de suporte externo que reforce as estratégias de coping da criança. A literatura da especialidade foca inúmeros sinônimos de resiliência (Anaut, 2005; Assis, Pesce, \& Avanci, 2006; Cyrulnik, 2003; Felgueiras, 2008; Pinheiro, 2004; Poletti \& Dobbs, 2007), como a capacidade para transcender circunstâncias difíceis do ambiente infantil para se tornar um adulto de sucesso, funcional, a partir da presença de figuras significativas e do estabelecimento de vínculos, em que o conjunto de mecanismos individuais e familiares mantêm a pessoa em relativa harmonia interior apesar de traumatismos vividos. Igualmente reenvia para a capacidade de reconstruir um continuum (que varia com as situações e as etapas do desenvolvimento) numa adaptação extraordinariamente boa diante de estresse grave e contínuo e retomando a pessoa estressada a um nível adaptativo. Ou seja, é a capacidade para sair vencedor (sair bem e socialmente aceite) de uma prova que poderia ter sido traumática e adaptado à situação adversa, recorrendo a recursos internos (intrapsíqui- 
cos) e externos (ambiente social e afetivo). No fundo é a manutenção de um processo normal de desenvolvimento apesar das condições difíceis, sendo algo sistemático, dinâmico e complexo, resultando da interação entre o indivíduo e o ambiente e sendo capaz de atribuir significado a experiências inicialmente sem sentido, de forma a elaborá-las e assim poder superá-las. É este constructo psicológico altamente complexo que a "The Resilience Scale", (originalmente de Wagnild, \& Young, 1993) propõe-se a medir (Wagnild \& Young, 1987, 1990, 1993): uma variável psicológica considerada, na literatura, como um potencial presente em cada indivíduo, desenvolvido em função do ciclo da vida, das fases do desenvolvimento psicológico e das circunstâncias ambientais. Em Portugal ainda não há uma escala adaptada para a população adulta, pelo que a adaptação dessa escala é pertinente.

\section{Versão original da escala}

A "The Resilience Scale,"na sua versão original, procura medir níveis de adaptação psicossocial positiva em situações e eventos de vida adversos através de 25 itens descritos de forma positiva com respostas tipo Likert, numa escala ordinal desde 1 (discordo totalmente) a 7 (concordo totalmente). Os valores totais são obtidos por somatório dos valores das respostas obtidas e podem variar entre 25 a 175, em que valores altos equivalem a elevada resiliência. Essa escala permite identificar o grau de resiliência individual enquanto característica positiva da personalidade e promotora da adaptação. Inicialmente as autoras encontraram 5 constituintes da resiliência: serenidade, perseverança, autoconfiança, sentido de vida e autossuficiência. No entanto as autoras encontraram ambiguidades na interpretação para 5, 4 e 3 fatores, ou seja, encontraram afinidades entre fatores, diminuindo-se os 5 fatores iniciais para dois fatores (critério de Kaiser). Assim, os 25 itens foram divididos em duas subescalas: uma subescala com 17 itens/Fator I: "Competência Pessoal" e uma subescala com 8 itens "Aceitação de Si Próprio e da Vida" (Fator II). O fator I sugere autoconfiança, independência, determinação, invencibilidade, mestria, fontes de recursos e perseverança (itens $1,2,3,4,5,6,9,10,13,14$, $15,17,18,19,20,23,24)$. O Fator II sugere adaptabilidade, equilíbrio, flexibilidade e uma perspetiva equilibrada da vida (itens $7,8,11,12,16,21,22$, 25). Ambos os fatores refletem a base teórica subjacente à resiliência contribuindo para a validade do constructo. Do ponto de vista psicométrico a escala original pode ser caracterizada da seguinte forma: fidelidade alta (coeficiente alpha de .91)/a correlação entre os itens variou entre 0,34 a 0,75 , com uma maioria entre 0,50 e 0,70 /todos mostraram-se significativos com pd"0,001/através da análise fatorial de componentes principais encontrou-se um eigenvalue para o fator I de 9,56 explicando 38,3\% da 
variância/os valores dos coeficientes variaram desde 0,3 a 0,76 com 23 dos 25 itens situados entre 0,45 e $0,76 /$ a escala original também apresenta validade na sua consistência interna: a sua consistência interna é aceitável ( $r=0,91$, pd"0,01 e vários estudos apontam para 0,76-0,91)/o teste reteste apresentou correlações aceitáveis, em 5 momentos diferentes, desde 0,67 a 0,84 (pd"0.01). Verificou-se igualmente que os escores mais altos de resiliência estavam associados a alta moral $(r=0,28)$, satisfação de vida $(r=0,30)$, melhor saúde física $(r=00,26)$ e a níveis mais baixos de depressão $(0,37)$. Foram encontradas 3 adaptações para a língua portuguesa: uma versão para a população brasileira (Pesce, Assis, \& Oliveira, 2004; Pesce et al 2005) com 997 adolescentes com idades entre os 12 e os 19 anos e com uma consistência interna de $a / p h a=.80$, um estudo preliminar (Vara \& Sani, 2006) com 380 adolescentes portugueses, com idades entre os 12 e os 18 anos e com um nível de consistência interna (alpha=.86), e uma adaptação e validação (Felgueiras, 2008) para adolescentes portugueses com idades entre os 10 e 16 anos e com valor alpha=.82. Nenhuma versão foi, até ao momento, alcançada para adultos portugueses.

\section{Método}

Quanto ao tipo de estudo, seguiu-se um desenho de investigação observacional com um método de amostragem probabilístico. Como procedimento começou-se por contactar as autoras do estudo preliminar (Vara \& Sani, 2006) e a autora da validação para pré-adolescentes/ adolescentes portugueses (Felgueiras, 2008) para pedido de informações. Seguidamente contactou-se as autoras da escala original solicitando autorização para a presente validação. Após a obtenção da autorização procedeu-se à retrotradução de forma a garantir, na versão adaptada, equivalência linguística e conceptual. Inicial e paralelamente procedeu-se à revisão da literatura. De forma a obter a equivalência linguística e conceptual foi sugerido pelas autoras da escala original uma retrotradução da versão obtida por Felgueiras (Felgueiras, 2008). Assim, os itens em língua portuguesa definidos por Felgueiras foram novamente traduzidos para a língua inglesa por duas psicólogas portuguesas com domínio da língua inglesa, procurando-se consensualidade e equivalência semântica. Uma delas não tinha conhecimento da escala original e das escalas em língua portuguesa. $O$ fato de terem sido duas psicólogas a retraduzir permitiu a consideração de aspectos clínicos/psicológicos inerentes, garantindo-se uma maior validade do conceito inerente a cada item, bem como uma maior validade de conteúdo. Os itens 2, 7, 21 da escala original foram sujeitos a duas retrotraduções de forma a preencherem os critérios e exigências linguísticas e conceituais exigidas pelas 
autoras enquanto os restantes itens foram sujeitos a apenas uma retrotradução. Durante esse processo foi possível a interação sistemática com uma das autoras da escala original (Dra. Gail Wagnild), que teceu alguns comentários decisivos. Garantiu-se que os itens traduzidos fossem equivalentes à versão original linguística e semanticamente e que as novas expressões fossem consideradas relevantes na cultura portuguesa. Optou-se, nessa fase, por manter o mesmo número de 25 itens, com 7 possibilidades de resposta, num regime de autoadministração, de forma a garantir uma maior equivalência operacional. Quanto à existência de um $26^{\circ}$ item "I am resilient" as autoras explicaram que este não faz parte da "The Resilience Scale", sendo apenas um item extra usado para contribuir para a validade da escala: o item 26 é correlacionado com o escore total da escala para melhorar a validade, não sendo necessário usá-lo com obrigatoriedade. Após a retrotradução receber um parecer favorável das autoras da escala original realizou-se um estudo piloto. A realização do estudo piloto $(n=20)$ permitiu verificar se os indivíduos entendiam as afirmações e estrutura do teste. Este pré-teste permitiu comprovar que as afirmações ( 25 itens) estavam perceptíveis sendo apenas necessário agregar aos números das respostas 2, 3, 4, 5, 6 uma denominação concreta desde o Discordo Muito ao Concordo Muito: isso porque inicialmente apenas o nível 1 e 7 estavam nominalmente discriminados (Discordo Totalmente/Concordo Totalmente).Todo esse processo linguístico e conceitual deu origem à tradução da escala que posteriormente foi submetida a análise estatística de forma a verificar a sua equivalência psicométrica e garantir que a nova versão mede apropriadamente (validade, fidelidade e sensibilidade) o conceito em estudo. Seguidamente, de forma a analisar a equivalência psicométrica da versão adaptada, procedeu-se à recolha da amostra. Os dados foram recolhidos em dois universos distintos: Instituto Superior Dom Afonso III em Loulé (maioritariamente) e Igreja Evangélica Batista de Faro. Depois de, individualmente, serem explicados os objetivos do estudo, o modo de preenchimento, bem como a garantia de voluntariedade e anonimato (consentimento informado), os indivíduos adultos, de ambos os sexos, preencheram o questionário, numa média de 4 minutos. Os mesmos foram recolhidos e posteriormente tratados no programa SPSS - Statistical Package for Social Sciences (versão 18.0). Após proceder-se à análise descritiva da amostra e da escala, calcularam-se os valores alpha de Cronbach (estimativa de confiabilidade dos resultados obtidos através de um instrumento, tendo em conta as decisões dos inquiridos e as variâncias associadas), reduzindo-se o número de itens da escala original e abrindo-se portas à análise fatorial. Na rotação ortogonal ou varimax (tal como no original), optou-se por parar a produção de fatores ao atingir $5 \%$ de variância porque se pretendeu comparar os resultados obtidos com os da escala original. Assim, 23 itens correlacionados foram agrupados em fatores que permitiram 
entender a estrutura da matriz de correlações e melhor interpretar os resultados. Optou-se por uma rotação ortogonal e não oblíqua exatamente porque a primeira é mais generalista, proporcionando uma solução de fatores mais ajustada à escala original, além de produzir dados similares à rotação oblíqua (que normalmente é usada em estudos exploratórios quando se pretende resultados que melhor se ajustem à amostra em estudo). Para avaliar a adequação dos dados a uma análise fatorial e de forma a assegurar que a análise em Componentes Principais pode ser realizada, duas condições foram asseguradas: adequabilidade e esfericidade. Para tal recorreu-se aos testes KMO (Kaiser-Meyer-Olkin) e Bartlett. O KMO indicou a proporção da variância dos dados que pode ser atribuída a um fator comum de todas as variáveis e o teste de esfericidade de Bartlett testou a esfericidade (Tabela 2). Em seguida passou-se à análise das comunalidades (a porção da variável que pode ser atribuída aos fatores comuns e é partilhada com todas as outras variáveis). A análise das comunalidades orientou a agregação de dados (arrumação dos itens em fatores). Seguidamente analisou-se os eigenvalues (permitiram calcular o número de fatores, eixos ou dimensões) em causa, sendo que conta o número de fatores com um Inicial Eigenvalue superior a 1. Seguidamente recorreu-se à análise da matriz das componentes com rotação varimax (Tabela 3) de forma a extremar os valores obtidos para que cada variável se associe a apenas 1 dos 4 fatores. Esse método, de rotação ortogonal, pretendeu que, para cada componente principal, existissem apenas alguns pesos significativos e todos os outros fossem próximos de zero, isto é, o objetivo foi maximizar a variação entre os pesos de cada componente principal, daí o nome varimax. Posteriormente e de forma a denominar cada fator recorreu-se à denominação inicialmente defendida por Wagnild e Young (1987, 1990, 1993): Perseverança (persistência na adversidade, vontade de continuar a reconstruir a vida mesmo lidando com problemas); Autoconfiança (crença no próprio e nas suas capacidades, reconhecendo as próprias limitações mas sendo capaz de depender de si mesmo); Serenidade (perspetiva equilibrada da vida, considerando uma ampla variedade de experiências e aceitando os acontecimentos, mesmo adversos, de forma serena e com capacidade de exercer autodisciplina); Autossuficiência (sentido de unicidade, consciência de que o percurso de vida de cada pessoa é único e que certas etapas são enfrentadas não em grupo mas em solidão) e Sentido de vida (noção de que se tem algo para que viver, de que a vida tem um sentido, uma razão). Através da transformação de variáveis e Visual Binning, calcularam-se os valores médios de corte para cada subescala. Ainda elaborou-se um teste reteste numa amostra de 32 indivíduos e salientaramse as conclusões finais. 


\section{Participantes}

A amostra foi composta por 365 indivíduos (sendo 43,5\% do gênero masculino e $56,5 \%$ do gênero feminino). Uma das limitações do estudo inerente à escala original é o fato de a mesma ter incluído apenas mulheres na sua amostra. Nesta investigação procurou-se ultrapassar essa limitação incluindo uma porcentagem favorável de indivíduos do sexo masculino. No que respeita às idades, a idade média situa-se nos 33,92 anos, a mediana situa-se nos 31,00 e a moda situa-se nos 32,00, com um mínimo de 18 anos e um máximo de 83 anos. 0 desvio padrão é 13,69 . Tendo em conta que cerca de $2,7 \%$ da amostra tem mais de 70 anos, procurou-se garantir que esses participantes reunissem condições cognitivas que lhes permitisse responder ao questionário com clara noção do real. No que diz respeito aos níveis de resiliência, nessa amostra encontra-se uma média de 132,44, com uma variância de 220,233 e um desvio padrão de 14,840, para 23 itens em análise. Os resultados revelam bons níveis de resiliência, tendo em conta que na escala original os escores variam entre 25 a 175 e nessa versão adaptada variam entre 25 e 161.

\section{Resultados}

De forma a obter a equivalência psicométrica calculou-se as médias e os desvios padrão para os itens definidos na escala e foi possível verificar que as médias situam-se entre 4,94 (Item 11) e 6,52 (Item 1), com um erro padrão entre 0,20 (Item 4 e Item 18) e 0,91 (Item 1). A variância situa-se entre 0,838 (Item 18) e 17,51 (Item 1). O desvio padrão situa-se entre 0,91 (Item 18) e 4,185 (Item 1). E a amplitude situa-se entre 1,912 (Item 15) e 7,44 (Item 7). De forma a analisar a homogeneidade calculouse as correlações entre o valor global e o alpha de Cronbach e os valores de cada item, estimando-se que o alpha de Cronbach para os 25 itens iniciais era mais baixo do que para 23 itens pelo que manteve-se 23 itens, excluindo-se os itens 1 e 7.0 valor alpha de Cronbach aumentou para 0,868 com escores a variar entre 25 e 161 (Tabela 1).

Tabela 1

Alpha de Cronbach para 23 itens

\begin{tabular}{ccc}
\hline $\begin{array}{c}\text { Cronbach's } \\
\text { Alpha }\end{array}$ & $\begin{array}{c}\text { Cronbach's Alpha Based on } \\
\text { Standardized Items }\end{array}$ & N of Items \\
\hline, 868 &, 880 & 23 \\
\hline
\end{tabular}


Tabela 2

Kaiser-Meyer-Olkin (KMO) e Teste de Bartlett

\begin{tabular}{ccc}
\hline Kaiser-Meyer-Olkin Measure of Sampling Adequacy. &, 893 \\
\hline Bartlett's Test of Sphericity & Approx. Chi-Square & 14104,255 \\
& df & 253 \\
Sig. &, 000 \\
\hline
\end{tabular}

Tabela 3

Análise da matriz das componentes com rotação varimax Rotated Component Matrix ${ }^{\mathrm{a}}$

\begin{tabular}{lllll}
\hline \multicolumn{5}{c}{ Component } \\
\hline & 1 & 2 & 3 & 4 \\
\hline Resiliência2 &, 425 &, 043 &, 179 &, 445 \\
Resiliência3 &, 213 &, 315 &, 006 &, 535 \\
Resiliência4 &, 292 &, 631 &,- 071 &, 261 \\
Resiliência5 &, 366 &, 198 &, 020 &, 439 \\
Resiliência6 &, 050 &, 725 &, 357 &, 083 \\
Resiliência8 &, 315 &, 329 &, 350 &, 259 \\
Resiliência9 &, 653 &, 120 &, 321 &, 011 \\
Resiliência10 &, 176 &, 572 &, 445 &, 011 \\
Resiliência11 &,- 016 &, 076 &, 403 &, 460 \\
Resiliência12 &,- 034 &, 083 &, 684 &, 204 \\
Resiliência13 &, 215 &, 046 &, 481 &, 137 \\
Resiliência14 &, 467 &, 418 &, 008 &,- 037 \\
Resiliência15 &, 129 &, 630 &, 502 &,- 209 \\
Resiliência16 &, 350 &, 452 &,- 033 &, 223 \\
Resiliência17 &, 649 &, 197 &, 201 &, 213 \\
Resiliência18 &, 475 &, 419 &, 064 &, 238 \\
Resiliência19 &, 573 &, 267 &,- 056 &, 155 \\
Resiliência20 &, 157 &, 117 &,- 049 &, 464 \\
Resiliência21 &, 075 &, 539 &,- 040 &, 351 \\
Resiliência22 &, 236 &, 047 &, 593 &,- 081 \\
Resiliência23 &, 721 &,- 009 &, 132 &, 169 \\
Resiliência24 &, 696 &, 174 &, 198 &, 212 \\
Resiliência25 &, 090 &,- 054 &, 312 &, 669 \\
\hline Extraction Method: Principal Component Analysis. & \\
Rotation Method:Varimax with Kaiser Normalization. & \\
\hline a. Rotation converged in 21 iterations. & & \\
\hline & & & & \\
\hline
\end{tabular}


A análise fatorial garantiu a validade de constructo reduzindo as 23 variáveis a uma dimensão menor, representando-as por meio de uma única variável estatística (variate) que expressa uma combinação linear das variáveis quantitativas originais. Neste estudo os dados apontam para um valor $\operatorname{KMO}(0,893)>0.5$ (os dados são adequados à análise fatorial, encontrando-se o valor muito próximo do valor 1 e estando no limite entre bom e muito bom) e quanto ao Bartlett verificou-se (Sig. 0,000) $<.05$ (os dados apresentam esfericidade e estão adequados a aplicação de análise fatorial, em componentes principais). Neste estudo as comunalidades iniciais são iguais a 1 e após a extração variam entre 0,384 e 0,715, indicando que todas elas podem fazer parte da análise (pois só se retirariam variáveis com valores, após a extração, inferiores a 0,3). Neste estudo encontraram-se, inicialmente, 6 componentes com um inicial eigenvalue superior a 1, sugerindo a existência de 6 fatores e explicando $56,660 \%$ da variância. No entanto, seguindo o procedimento da escala original optou-se por contemplar apenas os fatores que expliquem a variância total acima de $5 \%$, restando apenas 4 com $47,172 \%$ da variância explicada. Segundo o método varimax, como resultado inicial observou-se: Fator I $(9,17,19,23,24)$; Fator II $(4,6,10,15,21)$; Fator III $(12,22)$; Fator IV $(3,25)$. Sendo que 7 itens apresentaram loadings abaixo de $0,50 \%$, sugerindo necessidade de reavaliar a sua localização. Foram eles: $2,5,8,11,13,14,16$, 18,20 . Esses itens, com loadings abaixo de $0,50 \%$, foram posteriormente distribuídos pelos fatores nos quais apresentavam maior peso (ainda que abaixo de 0,50) e segundo a revisão teórica do construto (Tabela 4). E, tendo por base a revisão da literatura e os dados psicométricos, os 23 itens finais foram distribuídos por 4 fatores, nomeados da seguinte forma:

Fator I - Perseverança - remete para a entusiástica persistência em encontrar soluções para os problemas, vencendo adversidades. É a vontade de continuar a reconstruir a vida confiando em si mesmo e numa atitude de autodisciplina (Itens da escala final 7, 12, 15, 16, 17, 21, 22).

Fator II - Sentido de vida - remete para a consciência de que se tem algo significativo para que viver. Remete para a noção de que a vida tem um sentido (uma razão) na qual o indivíduo se foca evitando ficar obcecado com questões que não pode resolver. Envolve determinação e satisfação pelo alcance de objetivos definidos (Itens da escala final 3, 5, 8, 13, 14, 19). Fator III - Serenidade - remete para uma perspetiva equilibrada e focada nos propósitos da própria vida, com capacidade para aceitar a variedade de experiências (mesmo adversas) de forma serena e entusiástica e com capacidade de exercer a autoestima (Itens da escala final 6, 9, 10, 11, 20). Fator IV - Autossuficiência e autoconfiança - é o sentido de unicidade, é a consciência de que o percurso de vida de cada pessoa é único e que certas etapas são enfrentadas não em grupo mas em solidão, conseguindo a pessoa estar por conta própria e sendo capaz de depender essencialmente 
de si. Igualmente remete para crença no próprio, nas suas capacidades e o interesse pela própria vida, reconhecendo as próprias limitações e sendo capaz de depender de si mesmo (Itens da escala final 1,2,4, 18, 23).

Tabela 4

Distribuição dos itens pelos 4 fatores

\begin{tabular}{l}
\hline \multicolumn{2}{c}{ Segundo o Método Varimax } \\
\hline Fator I \\
9 - Sinto que consigo lidar com várias \\
coisas ao mesmo tempo. \\
17 - A confiança em mim próprio/a \\
ajuda-me a lidar com tempos difíceis. \\
19 - Normalmente consigo olhar para \\
uma situação de várias perspetivas. \\
23 - Quando estou numa situação \\
difícil, normalmente consigo \\
encontrar uma solução. \\
24 - Tenho energia suficiente para \\
fazer o que deve ser feito.
\end{tabular}

4 - Manter-me interessado/a nas 4 - Manter-me interessado/a nas atividades do

atividades do dia a dia é importante dia a dia é importante para mim. para mim.

6 - Sinto-me orgulhoso/a por ter alcançado objetivos na minha vida.

10 - Sou determinado/a.

15 - Mantenho-me interessado/a nas coisas.

Versão Final
Perseverança
9 - Sinto que consigo lidar com várias coisas ao
mesmo tempo.

14 - Tenho autodisciplina

17 - A confiança em mim próprio/a ajuda-me a

lidar com tempos difíceis.

19 - Normalmente consigo olhar para uma

situação de várias perspetivas.

23 - Quando estou numa situação difícil normalmente consigo encontrar uma solução.

24 - Tenho energia suficiente para fazer o que deve ser feito.

18 - Numa emergência, sou alguém com quem geralmente as pessoas podem contar. Sentido de Vida

6 - Sinto-me orgulhoso/a por ter alcançado

objetivos na minha vida.

10 - Sou determinado/a.

15 - Mantenho-me interessado/a nas coisas.

21 - A minha vida tem um propósito.

Fator III

21 - A minha vida tem um propósito.

16 - Geralmente consigo encontrar algo que me

12 - Vivo um dia de cada vez faça rir.

12 - Vivo um dia de cada vez

22 - Eu não fico obcecado/a com 22 - Eu não fico obcecado/a com coisas que não coisas que não posso resolver posso resolver

8 - Sou amigo/a de mim próprio/a./.

11 - Raramente me questiono se a vida tem sentido.

13 - Posso passar por tempos difíceis porque enfrentei tempos difíceis antes.

\begin{tabular}{|c|c|}
\hline $\begin{array}{l}\text { Fator IV } \\
3 \text { - Sou capaz de depender de mim } \\
\text { próprio/a mais do que de qualquer } \\
\text { outra pessoa. } \\
25 \text { - Não tenho problema com o facto } \\
\text { de haver pessoas que não gostam de } \\
\text { mim }\end{array}$ & $\begin{array}{l}\text { Autossuficiência e autoconfiança } \\
3 \text { - Sou capaz de depender de mim próprio/a mais } \\
\text { do que de qualquer outra pessoa. } \\
25 \text { - Não tenho problema com o facto de haver } \\
\text { pessoas que não gostam de mim } \\
2 \text { - Normalmente eu lido com os problemas de } \\
\text { uma forma ou de outra } \\
5 \text { - Posso estar por conta própria se for preciso. } \\
20 \text { - Por vezes obrigo-me a fazer coisas quer } \\
\text { queira quer não. }\end{array}$ \\
\hline
\end{tabular}

Itens sujeitos a revisão (posterior distribuição pelos fatores): 2 - Normalmente eu lido com os problemas de uma forma ou de outra./ 5 - Posso estar por conta própria se for preciso./ 8 - Sou amigo/a de mim próprio/a./. 11 - Raramente me questiono se a vida tem sentido./ 13 - Posso passar por tempos difíceis porque enfrentei tempos difíceis antes. /14 - Tenho autodisciplina./ 16 - Geralmente consigo encontrar algo que me faça rir./ 18 - Numa emergência, sou alguém com quem geralment 
Os valores do coeficiente alpha de Cronbach revelaram-se satisfatórios para cada fator: .839 (Fator I); .814 (Fator II); .604 (Fator III); .672 (Fator IV). Salientando-se que um reduzido número de itens em cada fator contribui para a diminuição do valor alpha de Cronbach, e tendo em conta que tal acontece nessa escala, consideram-se os valores alpha obtidos satisfatórios. De forma a avaliar a sensibilidade dos itens recorreu-se à análise do coeficiente de assimetria e do coeficiente de achatamento. Neste estudo o coeficiente de assimetria (Sk), para cada item, não ultrapassou os 3 valores absolutos e o coeficiente de achatamento $(\mathrm{Ku})$, para cada item, não ultrapassou os 10 valores absolutos, indicando sensibilidade dos itens. Igualmente procurou-se verificar a aderência à normalidade dos itens através do teste de Kolmogov-Smirnov de aderência à normalidade. Esse teste permitiu analisar o ajustamento/aderência à normalidade da distribuição de cada variável/item, através da comparação das frequências relativas acumuladas observadas com as frequências relativas esperadas. $O$ valor do teste remeteu para a maior diferença entre essas frequências (relativas acumuladas observadas versus relativas esperadas). Para um nível de significância de 0,05 , neste estudo, o teste de Kolmogorov-Smirnov apontou para uma distribuição não normal para cada item, pois cada um deles apresentou um nível de significância inferior a 0,05: rejeita-se HO. No entanto, quando os dados foram sujeitos a uma estatística não paramétrica verificou-se uma distribuição normal para cada variável. Quanto ao escore total da escala, o teste de KolmogorovSmirnov indicou a presença de uma distribuição não normal (Sigd"0,05), comprovado pelo histograma de frequências (no qual as observações da variável RTotal são organizadas em classes e a frequência de cada classe é representada). A média situa-se em 5,76, com um desvio padrão de 0,645 , um mínimo de 1,70 e um máximo de 7,00. De forma gráfica observou-se no Normal Q-Q Plot of Total uma certa tendência para a normalidade ainda que incompleta, e no gráfico Detrended Normal Q-Q Plot of Total verificou-se a presença de efetivos resíduos na amostra, responsáveis pela ausência de normalidade na distribuição. Para corrigir essa ausência de normalidade na distribuição ter-se-ia de recorrer a uma outra amostra ou, talvez, aumentar essa mesma. No entanto, quando a variável foi sujeita a uma estatística não paramétrica verificou-se a presença de uma distribuição normal, tal como nos itens isolados. A ausência de normalidade na distribuição reenviou para a necessidade de recorrer ao uso de testes não paramétricos (menos potentes do que os paramétricos). No que diz respeito ao método teste-reteste (avaliou o índice de fidedignidade do instrumento) recorreu-se a uma amostra de 32 indivíduos, de forma a reaplicar o instrumento 4 semanas após a primeira aplicação. Verificouse para o primeiro momento do teste um valor alpha de Cronbach $=.913$, uma média $=114,06$, uma variância $=457,673$ e um desvio padrão $=$ 21,810 . Para o segundo momento (reteste) um valor alpha de Cronbach $=$ 
.898 ; uma média $=120.41$, uma variância $=357,281$ e um desvio padrão $=$ 18,902 . Verificou-se que não se encontram diferenças significativas nos valores alpha de Cronbach entre os dois momentos (a diferença entre ambos é apenas de 0,015). Ainda no método reteste verificou-se que, nessas duas amostras emparelhadas, a maioria das variáveis não apresenta distribuição normal (no primeiro momento a distribuição não é normal para os itens $2,4,5,6,8,9,10,12,14,15,16,20,21,23,24,25$ e no segundo momento não é normal para os itens $2,4,5,6,8,10,11,12,14,15$, $16,17,18,19,20,21,22,23,24,25)$. Pelo que, quando se testou a igualdade de médias, optou-se pelo teste Wilcoxon (não paramétrico) para variáveis ordinais, sendo a hipótese de partida: $\mathrm{HO}: E(X)=E(Y)$. Sendo a hipótese nula aceite e comprovando-se que não há diferenças estatísticas entre as médias do primeiro e do segundo momento de observação da mesma amostra. Ainda, segundo o método reteste, recorreu-se ao Teste de Spearman para verificar, numa análise bivariada, a correlação entre os dois momentos. O Teste de Spearman (não paramétrico) avaliou a correlação de ordem (em variáveis ordinais) e no caso deste estudo verificou-se que o resultado de Correlation Coefficient é igual a 1,000, sugerindo que há associação direta entre variáveis, ou seja, rejeita-se $\mathrm{H} 0$ e comprova-se a boa estabilidade temporal da escala. Ainda nessa amostra (através do Visual Binning) calcularam-se os valores médios de corte para cada subescala, sendo os seguintes: Sub-Escala Perseverança (Perseverança Baixa 1,00-5,51; Perseverança Média 5,72-6,14; Perseverança Alta 6,15+); Subescala Sentido de Vida (Sentido de Vida Baixo 1,00-5,83; Sentido de Vida Médio 5,84 - 6,33; Sentido de Vida Alto 6,34+); Subescala Serenidade (Baixo 1,00-5,00; Médio 5,01 - 5,80; Alto 5,81+); Subescala Autossuficiência e Autoconfiança (Baixa 1,00 - 5,40; Média 5,41-6,00; Alta 6,01+). No entanto, convém recordar que a resiliência não é estanque ou linear em cada pessoa: a mesma pode variar ao longo da vida e de acordo com as várias situações às quais o indivíduo é sujeito. Adiante é possível aceder à escala final. 
Quadro

Versão Final da Escala de Resiliência adaptada para População Adulta Portuguesa

\section{Por favor, leia as instruções.}

Encontrará sete números, variando de "1" (Discordo Totalmente) a "7" (Concordo Totalmente). Assinale com uma cruz (X) o número que melhor indica seus sentimentos sobre essa afirmação. Por exemplo, se você discordar totalmente com a afirmação, assinale "1". Se você é neutro, assinale "4" e se concordar totalmente, assinale "7".

Identificação (facultativo)

\section{Idade:}

Sexo:

1 Normalmente eu lido com os problemas de uma forma ou de outra.

2 Sou capaz de depender de mim próprio/a mais do que de qualquer outra pessoa.

3 Manter-me interessado/a nas atividades do dia a dia é importante para mim.

4 Posso estar por conta própria se for preciso.

5 Sinto-me orgulhoso/a por ter alcançado objetivos na minha

vida.

6 Sou amigo/a de mim próprio/a.

7 Sinto que consigo lidar com várias coisas ao mesmo tempo.

8 Sou determinado/a.

9 Raramente me questiono se a vida tem sentido.

10 Vivo um dia de cada vez.

11 Posso passar por tempos difíceis porque enfrentei tempos

difíceis antes.

12 Tenho autodisciplina.

13 Mantenho-me interessado/a nas coisas.

14 Geralmente consigo encontrar algo que me faça rir.

15 A confiança em mim próprio/a ajuda-me a lidar com tempos difíceis.

16 Numa emergência, sou alguém com quem geralmente as pessoas podem contar.

17 Normalmente consigo olhar para uma situação de várias perspetivas.

18 Por vezes obrigo-me a fazer coisas quer queira quer não.

19 A minha vida tem um propósito.

20 Eu não fico obcecado/a com coisas que não posso resolver.

21 Quando estou numa situação difícil, normalmente consigo

encontrar uma solução.

22 Tenho energia suficiente para fazer o que deve ser feito.

23 Não tenho problema com o facto de haver pessoas que não

gostam de mim.

\section{Conclusão}

Numa sociedade pós-moderna em constante mudança e exigindo constante adaptação dos seus intervenientes, faz todo o sentido a Psicologia continuar a investigar a resiliência. Através deste trabalho procurou-se a adaptação da "The Resilience Scale" para a população adulta portuguesa, por constituir uma lacuna na avaliação psicológica em Portugal. Obteve-se equivalência linguística e conceptual através do processo de retrotradução e garantiu-se a validade do conceito inerente a cada 
item, bem como a validade de conteúdo. Os itens traduzidos são equivalentes à versão original linguística e semanticamente, com expressão relevante na cultura portuguesa. A equivalência operacional foi garantida. Quanto à validação psicométrica $(\mathrm{n}=365)$ salienta-se o alpha de Cronbach de 0,868 para 23 itens (com remoção dos itens iniciais 1 e 7), sugerindo boa consistência interna, fiabilidade estável e homogeneidade. Esse valor está próximo do valor apresentado pela escala original (0.91) e acima do valor apresentado pela escala aferida para adolescentes portugueses (Felgueiras, 2008), igual a 0,82. O fato de esse instrumento gerar valores alpha altos em diferentes adaptações permite concluir que a estimativa de fiabilidade dos dados obtidos é alta, pois informa sobre a precisão do instrumento através do seu uso repetido, em diferentes amostras, e comprova a validade do processo inferencial. Quanto aos escores, encontrouse um valor médio de 132.44, revelando níveis bons de resiliência. A análise fatorial garantiu a validade de construto, reduzindo as 23 variáveis a uma dimensão menor (verificando-se adequabilidade, esfericidade e comunalidade). Emergiram 4 fatores com $47.172 \%$ da variância explicada e pelos quais as 23 variáveis foram distribuídas através do método varimax, com valores alpha de Cronbach satisfatórios para cada um. Verificou-se a sensibilidade dos itens através da análise do coeficiente de assimetria e do coeficiente de achatamento. A estabilidade temporal da escala foi garantida ainda que a resiliência não seja estanque e podendo variar ao longo da vida. Como limitação salienta-se (tal como afirmam Wagnild \& Young) o fato dessa escala ser sensível à resiliência enquanto aspecto positivo de adaptabilidade mas não ser tão sensível a valores mais baixos desse construto: ou seja, empiricamente não é tão sensível a captar os aspectos teóricos da baixa resiliência e nem níveis mais baixos da mesma, pelo que seria interessante rever essa situação em futuros trabalhos.

Texto escrito conforme o Acordo Ortográfico - convertido pelo Lince.

Adaptation of "The Resilience Scale" for the adult population of Portugal

\begin{abstract}
This paper describes the adaptation of "The Resilience Scale" for Portuguese adults. This instrument was submitted to back-translation (linguistic and conceptual equivalence), to psychometric validation $(n=365)$, to pretest and retest. It was obtained conceptual and linguistic equivalence/psychometric validation with alpha Cronbach 0.868 for the 23 items, suggesting internal consistency. Factorial analysis grouped the 23 variables in 4 factors: I - Perseverance; II-Sense of life; III-Serenity; IV-
\end{abstract}


Self-reliance and self-confidence, with satisfactory alpha Cronbach values for each factor. The sensitivity of items was verified by the coefficient of asymmetry and flatness. It was proved temporal stability of the scale. The scale shows good psychometric characteristics checking levels of resilience in this population.

Keywords: Resilience. Test adaptation (Portugal). Adults.

\section{Adaptation du "The Resilience Scale" pour la population adulte portugaise}

Résumé:Cet article décrit l'adaptation du“The Resilience Scale" pour Portugais adultes. S'est procédée à la retraduction et à la validation psicométrique. Il s'est fait pré-teste et reteste. II s'est obtenu équivalence linguistique/conceptuelle et validation psicométrique avec alpha de Cronbach 0.868 pour 23 items, en suggérant consistance interne. L'analyse factorielle a regroupé 23 variables dans 4 facteurs: I - Persévérance; II - Sens de vie; III - Sérénité; IV - Autosuffisance et autoconfiance, avec des valeurs alpha de Cronbach satisfaisante pour chaque facteur. Il s'est vérifié de la sensibilité des items à travers le coefficient d'asymétrie et de la platitude et s'est vérifié la stabilité séculière. L'échelle révèle de bonnes caractéristiques psicométriques en examinant les niveaux de résilience dans cette population.

Mots-clés: Résilience. Test de adaptation (Portugal). Adultes.

\section{Adaptación de la "Escala de Resiliencia" para el adulto portugués}

Resumen: Este artículo describe la adaptación de la "Escala de la Resiliencia" para el adulto portugués. La escala fue sometida a retradición (equivalencia lingüística y conceptual), a validación psicométrica ( $n=365)$, a preprueba y reteste. no consiguió equivalencia lingüística/conceptual y validación psicométrica con alpha de Cronbach 0.868 para el 23 variables, sugiriendo consistencia interna. El análisis fatorial agrupó 23 variables en 4 fatores: I - Perseverencia; II - Sentido de vida; III - Serenidad; IV Autosuficiencia y autoconfianza, con valores alpha de Cronbach satisfactorios. La sensibilidad (coeficiente del achatamiento y asimetría) fue verificada. La estabilidad secular de la escala es buena. La escala divulga buenas características psicométricas y examina niveles de resistencia en esta población.

Palabras clave: Resiliencia. Adaptación de test (Portugal). Adultos. 


\section{Referências}

Anaut, M. (2005). A resiliência - ultrapassar os traumatismos. Lisboa: Climepsi.

Assis, S. G., Pesce, R. P., \& Avanci, J. Q. (2006). Resiliência: enfatizando a proteção dos adolescentes. Porto Alegre: Artmed.

Cyrulnik, B. (2003). Resiliência - essa inaudita capacidade de construção humana. Lisboa: Instituto Piaget.

Felgueiras, M. (2008). Adaptação e validação da Resilience Scale de Wagnild e Young para a cultura portuguesa. Porto: Universidade Católica Portuguesa.

Gonçalves, M. J. (2003). Aumentar a resiliência das crianças vítimas de violência. Análise Psicológica, 1(21), 23-30.

Pesce, R. P., Assis, S. G., \& Oliveira, R. V. C. (2004). Risco e proteção: em busca de um equilíbrio promotor de resiliência. Psicologia: Teoria e Pesquisa, 20(2), 135143.

Pesce, R.P., Assis, S., Avanci, J. Q., Santos, N. C., Malaquias, J.V., \& Carvalhaes, R. (2005). Adaptação transcultural, confiabilidade e validade da escala de resiliência. Caderno Saúde Pública, 21(2), 436-448.

Pinheiro, D. P. (2004). A resiliência em discussão. Psicologia em Estudo, 9(1), 67-75.

Poletti, R., \& Dobbs, B. (2007). A resiliência - a arte de dar a volta por cima. Petrópolis, RJ: Vozes.

Wagnild, G., \& Young, H. (1987). The Resilience Scale ${ }^{T M}$ Homepage. Recuperado em 21 de novembro de 2010, de www.resiliencescale.com

Wagnild, G. M., \& Young, H. (1990). Resilience among older women. Journal of Nursing Scholarship, 22, 252-255.

Wagnild, G. M., \& Young, H. (1993). Development and psychometric evaluation of the Resilience Scale. Journal of Nursing Measuremen, 2(1), 165-178.

Vara, M., \& Sani, A. (2006). Escala de resiliência de Wagnild \& Young: estudo preliminar de validação. In Atas da XI Conferência Internacional de Avaliação Psicológica: formas e contextos (pp. 333-340). Braga: Psiquilibrios. 
Cláudia Alexandra Ferreira de Carvalho Ng Deep, Instituto Superior Dom Afonso III. Endereço para correspondência: Convento Espírito Santo, 8100-641. Loulé, Portugal. Endereço eletrônico: claudiangdeep@gmail.com

Isabel Pereira Leal, Instituto Superior de Psicologia Aplicada. Endereço para correspondência: Rua Jardim do Tabaco, 34, 1149-041 Lisboa, Portugal. Endereço eletrônico: isabel.leal@iol.pt 